\title{
Modulation of distal colonic epithelial barrier function by dietary fibre in normal rats
}

\author{
J M Mariadason, A Catto-Smith, P R Gibson
}

University of

Melbourne,

Department of

Medicine, Royal

Melbourne Hospital,

Victoria 3050,

Australia

J M Mariadason

P R Gibson

\section{Department of Gastroenterology and Clinical Nutrition, Royal Children's Hospital, Victoria, Australia \\ A Catto-Smith \\ Correspondence to: Associate Professor P R Gibson.}

Accepted for publication 23 September 1998

\begin{abstract}
Background-Dietary fibre influences the turnover and differentiation of the colonic epithelium, but its effects on barrier function are unknown.

Aims-To determine whether altering the type and amount of fibre in the diet affects paracellular permeability of intestinal epithelium, and to identify the mechanisms of action.

Methods-Rats were fed isoenergetic low fibre diets with or without supplements of wheat bran $(10 \%)$ or methylcellulose $(10 \%)$, for four weeks. Paracellular permeability was determined by measurement of conductance and ${ }^{51} \mathrm{Cr}$-EDTA flux across tissue mounted in Ussing chambers. Faecal short chain fatty acid (SCFA) concentrations were assessed by gas chromatography, epithelial kinetics stathmokinetically, and mucosal brush border hydrolase activities spectrophotometrically.

Results-Body weight was similar across the dietary groups. Conductance and ${ }^{51} \mathrm{Cr}-\mathrm{EDTA}$ flux were approximately $25 \%$ higher in animals fed no fibre, compared with those fed wheat bran or methylcellulose in the distal colon, but not in the caecum or jejunum. Histologically, there was no evidence of epithelial injury or erosion associated with any diet. The fibres exerted different spectra of effects on luminal SCFA concentrations and $\mathrm{pH}$, and on mucosal indexes, but both bulked the faeces, were trophic to the epithelium, and stimulated expression of a marker of epithelial differentiation.

Conclusions-Both a fermentable and a non-fermentable fibre reduce paracellular permeability specifically in the distal colon, possibly by promoting epithelial cell differentiation. The mechanisms by which the two fibres exert their effects are likely to be different.

(Gut 1999;44:394-399)
\end{abstract}

Keywords: colon; differentiation; epithelium; fibre; paracellular permeability; proliferation

The colonic epithelium performs an essential barrier function by acting to prevent proinflammatory macromolecules from gaining access to the internal milieu. Macromolecules that permeate the epithelium do so mainly via the paracellular pathway, for which the tight junction is the rate determining structure. Evidence from human disease, including ulcerative colitis ${ }^{1}$ and Crohn's disease, ${ }^{2}$ and experi- mental animals ${ }^{3}$ suggests an association between the presence of intestinal mucosal inflammation and increased paracellular permeability. Whether impaired barrier function is the cause or effect (or both) of the mucosal inflammation is not known but impaired epithelial barrier function could lead to increased permeation of luminal proinflammatory macromolecules into the lamina propria, a process of potential pathogenic significance.

Despite the physiological importance of the maintenance of paracellular permeability in the colon, there are no published studies on whether variations in diet exert any influence on the permeability of the colonic epithelium. Some attention has been paid to the effect of dietary manipulation on small intestinal permeability. For example, removal of specific dietary macro- and micronutrients, ${ }^{45}$ total parenteral nutrition, ${ }^{6}$ and fasting ${ }^{7}$ are reported to increase paracellular permeability in rat small intestine. The colon, however, has a different relation to diet than does the small intestine, in that the interaction of dietary factors with the luminal flora may exert more influence than the dietary components themselves. Fermentation of dietary undigestible carbohydrate (such as fibre) and unabsorbed protein yields products such as short chain fatty acids (SCFAs), phenols, and reducing sulphur compounds, all of which may have beneficial or detrimental effects on the epithelium (reviewed by $\mathrm{Gibson}^{8}$ ). Minimisation of luminal fermentation by the unphysiological surgical procedure of diverting the faecal stream from a segment of colon in rats causes notable atrophy of the diverted segment and is associated with increased paracellular permeability. ${ }^{9}$ The mechanism for such an effect was not examined.

Of the fermentation products produced in the colonic lumen, only SCFAs have been investigated for their effects on paracellular permeability. In vitro, butyrate, acetate, and propionate all exert a concentration dependent reduction in paracellular permeability in the Caco-2 model of colonic epithelium, an effect that is maximal 48 hours after initial exposure. ${ }^{10}$ In contrast, acute exposure to butyrate increases paracellular permeability in guinea pig or rat colonic mucosa mounted in Ussing chambers (Mariadason et al, manuscript submitted). ${ }^{11}$ Luminal concentrations of SCFAs are readily amenable to modulation by diet. Altering the amount and type of dietary

Abbreviations used in this paper: $\mathrm{CCH}$, crypt column height; DPPIV, dipeptidylpeptidase IV; MI, mitotic index; PD, potential difference; SCFA, short chain fatty acid. 
carbohydrate alters colonic SCFA levels. ${ }^{12}$ Furthermore, diets high in fermentable fibre or starch resistant to digestion not only increase colonic SCFA levels but also are able to alter physiological indexes such as epithelial turnover, ${ }^{13}{ }^{14}$ and to alter disease pathogenesis such as tumour formation following carcinogen administration. ${ }^{14}{ }^{15}$ Limited indirect ${ }^{15}$ and direct data ${ }^{16}$ suggest that SCFAs are causally related to some of these effects, particularly tumour formation.

The present study aimed to determine whether altering the amount and type of fibre in the diet of normal rats influenced paracellular permeability in the distal colon. Wheat bran was chosen because it has previously been shown to increase notably distal colonic concentrations of SCFAs. ${ }^{12}$ As wheat bran is slowly fermented and considerable unfermented fibre remains in the faeces, the effect of a non-fermentable fibre, methylcellulose, which is associated with very low SCFA levels in the colonic lumen, ${ }^{13}$ was also examined. Relations between changes in permeability with changes in morphology, epithelial turnover and differentiation, and luminal indexes were also sought.

\section{Methods}

ANIMAL HUSBANDRY

Male Sprague-Dawley rats weighing approximately $160 \mathrm{~g}$ were obtained from the Monash University Animal Services, Victoria, Australia. Animals were housed at the Royal Melbourne Hospital Animal facility, with diurnal lighting, and access to food and water provided ad libitum. Animals were housed in groups of four, in drop bottom wire cages to reduce coprophagy. Rat diets were based on the AIN-76 standard for purified diets for rats and mice, ${ }^{18}$ and prepared as previously described. ${ }^{17}$ For all dietary intervention studies, the feeding period was four weeks and each dietary group comprised 12 rats. Studies were approved by the Hospital Campus Animal Ethics Committee of the Royal Melbourne Hospital. The conduct was in accordance with the guidelines laid down in the Australian Code of Practice for the Care and Use of Animals for Scientific Purposes in a registered animal facility.

MEASUREMENT OF BODY WEIGHT, FOOD CONSUMPTION, AND LUMINAL INDEXES

The rats were monitored daily regarding their general health and signs of stress, and were weighed weekly. Twenty four hour food consumption, 24 hour faecal output (wet weight), and faecal and caecal $\mathrm{pH}$ were measured as previously described. ${ }^{13}$ SCFA concentrations were determined in rat faecal and caecal contents by gas chromatography, as previously described. ${ }^{10}$

MEASUREMENT OF PARACELLULAR PERMEABILITY For measurement of paracellular permeability, animals were anaesthetised with Nembutal (Boehringer Ingelheim, Artarmon, New South Wales, Australia); two segments of distal colon (approximately 1 and $4 \mathrm{~cm}$ from the rectum), and one segment from each of the caecum and jejunum ( $5 \mathrm{~cm}$ distal to the ligament of Trietz) were removed, and the animals were sacrificed under anaesthesia. The distal colon and jejunum were stripped of the underlying muscle layers, and mounted in $0.76 \mathrm{~cm}^{2}$ Ussing chambers (CSIRO, Victoria, Australia). Due to difficulties in stripping, the caecum was mounted in Ussing chambers with the muscle layers intact. The apical and basolateral surfaces were bathed by separate reservoirs, each containing $15 \mathrm{ml} \mathrm{Krebs}$ buffer at $37^{\circ} \mathrm{C}$ and $\mathrm{pH}$ 7.4. The buffer contained $\mathrm{Na} 140 \mathrm{mM}$, $\mathrm{K} 10 \mathrm{mM}, \mathrm{Mg} 1.1 \mathrm{mM}$, Ca $1.25 \mathrm{mM}, \mathrm{Cl} 127.7$ $\mathrm{mM}, \mathrm{H}_{2} \mathrm{PO}_{4} 2 \mathrm{mM}, \mathrm{HCO}_{3}^{-} 25 \mathrm{mM}$, and glucose $10 \mathrm{mM}$ (BDH Chemicals, Poole, UK). Carbogen was bubbled through the chambers to oxygenate the tissue. A 30 minute equilibration period was allowed to elapse prior to commencement of measurement of paracellular permeability. Prior studies had shown this to be sufficient time for stabilisation of electrical indexes to occur. For measurement of transepithelial conductance, the spontaneous potential difference (PD) across the epithelium was determined, and the tissue clamped at zero voltage by introducing an appropriate short circuit current (Isc) with an automatic voltage clamp (DVC 1000; World Precision Instruments, New Haven, Connecticut, USA). The Isc was continuously monitored and PD measured by briefly removing the voltage clamp for 5-10 seconds every 15 minutes. Values at the end of the first, second, and third 15 minute periods were recorded. Transepithelial conductance $(\mathrm{G})$ was calculated according to Ohm's law, and expressed as $\mathrm{mS} / \mathrm{cm}^{2}$. For measurement of the transepithelial flux of EDTA, $2 \mathrm{Mbq}$ of ${ }^{51} \mathrm{Cr}$-EDTA (Australian Radioisotopes, New South Wales, Australia) was added to the apical reservoir at the end of the 30 minute equilibration period, and serial aliquots of $0.5 \mathrm{ml}$ and $2 \mathrm{ml}$, were taken at regular intervals from the apical and basolateral reservoirs respectively. Radioactivity was determined by gamma counting, and results expressed as rate coefficients in $\mu 1 / \mathrm{min} .{ }^{19} \mathrm{Occa}-$ sionally, the agar bridges would become damaged due to wear but were readily repaired. However, minor spills of EDTA across the bridge invalidated the EDTA flux results for those experiments and these were subsequently omitted from the analyses. In preliminary experiments, administration of vincristine to a group of eight rats three hours prior to sacrifice had no effect on paracellular permeability compared with a group of eight rats receiving vehicle only (data not shown).

MEASUREMENT OF COLONIC EPITHELIAL CELL KINETIC INDEXES

Cell proliferation was assessed by the vincristine sulphate (David Bull Laboratory, Melbourne, Australia) method of counting metaphase arrests, as previously reported in detail. ${ }^{13}$ Ten longitudinally sectioned crypts were evaluated by one investigator (JMM), blinded to the origin of the sections. Crypt column height $(\mathrm{CCH})$ was calculated as the mean number of cells per crypt column, determined by counting under light microscopy. ${ }^{13}$ The rate 

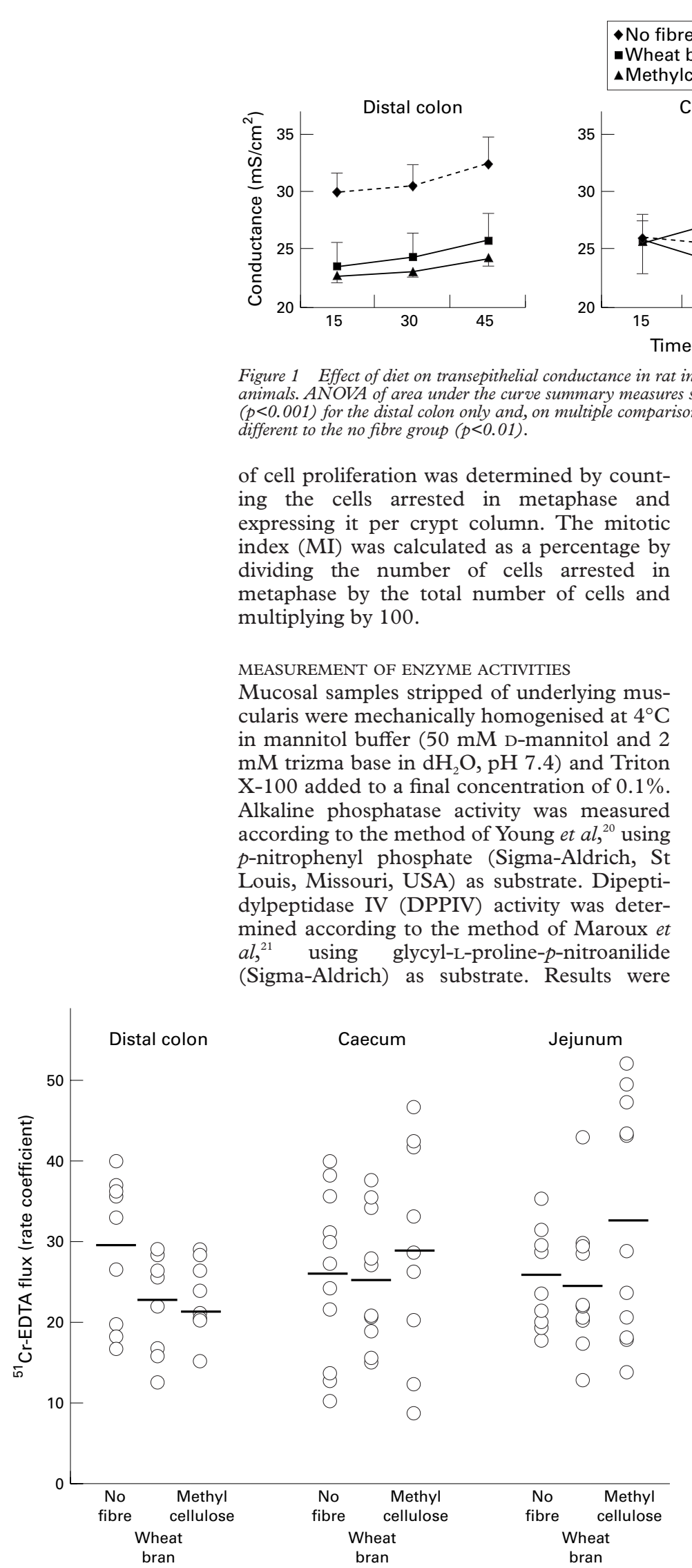

Figure 2 Effect of diet on the flux of ${ }^{51} \mathrm{Cr}$-EDTA across distal colon, caecum, and jejunum mounted in Ussing chambers. The diets contained no fibre, wheat bran, or methylcellulose. For the distal colon only, differences were statistically significant across the dietary groups $(p=0.004 ; A N O V A)$. EDTA flux in the wheat bran and methylcellulose groups was significantly different to that in the no fibre group on multiple comparisons $(p<0.05)$. expressed relative to cellular protein content, measured using bovine gamma globulin as standard. $^{22}$ The coefficient of variation of all assays was less than $10 \%$.

STATISTICAL METHODS

Group data from all experiments are expressed as mean (SEM). The results across dietary groups were compared by analysis of variance (ANOVA). A p value of not more than 0.05 was considered statistically significant. All statistical analyses were performed using Minitab for Windows, version 9.2 (Minitab Inc., State College, Pennsylvania, USA).

\section{Results}

EFFECT OF DIET ON ANIMAL HEALTH

All rats remained healthy and showed no evidence of stress during the duration of the experimental period. Gain in body weight after four weeks was similar across all dietary groups. Those fed a no fibre diet gained 182 (9) g, those fed wheat bran 165 (7) g, and those fed methylcellulose 177 (6) g. Twenty four hour food consumption, measured at the end of the fourth week, was highest in the wheat bran (35 (4) g/rat) and methylcellulose fed groups (31 (1) g/rat), and least in the energy dense no fibre diet (26 (9) g/rat).

\section{EFFECT OF DIET ON PARACELLULAR} PERMEABILITY

As fig 1 shows, transepithelial conductance in the caecum or jejunum was similar across the three diets. However, significant differences were observed across the diets in the distal colon ( $p=0.004$, ANOVA using area under the curve summary measure), due to the high conductance in rats fed a no fibre diet. Mean conductance was $24 \%$ less in both the wheat bran and methylcellulose groups.

The transepithelial flux of ${ }^{51} \mathrm{Cr}$ EDTA paralleled that of conductance. As fig 2 shows, the flux differed across the dietary groups in the distal colon $(p=0.004)$ but not caecum or jejunum. The higher flux in animals fed the no fibre diet was responsible for this difference. Mean flux was $23 \%$ higher than in the wheat bran group and $28 \%$ higher than in the methylcellulose group. 
Table 1 Effect of diet on faecal indexes

\begin{tabular}{|c|c|c|c|c|}
\hline & No fibre & Wheat bran & Methylcellulose & $p$ Value * \\
\hline 24 hour faecal output (g/rat) & $0.6(0.1) \dagger$ & $6.0(0.3)$ & $4.5(0.2)$ & $<0.001$ \\
\hline Faecal pH & $7.1(0.1) \ddagger$ & $5.7(0.1)$ & $6.9(0.1)$ & $<0.001$ \\
\hline Caecal pH & $6.9(0.1)$ & $6.5(0.1)$ & $7.2(0.6)$ & $<0.001$ \\
\hline \multicolumn{5}{|c|}{ Faecal SCFA ( $\mu \mathrm{mol} / \mathrm{g}$ faecal water) } \\
\hline Total & $17.7(1.9)$ & $29.8(4.1)$ & $3.2(0.7)$ & $<0.001$ \\
\hline Butyrate & $2.1(0.3)$ & $3.8(0.7)$ & $0.9(0.2)$ & $<0.001$ \\
\hline \multicolumn{5}{|c|}{ Caecal SCFA ( $\mu \mathrm{mol} / \mathrm{g}$ caecal water) } \\
\hline Total & $22.3(3.9)$ & $30.2(2.1)$ & $13.0(1.6)$ & $<0.001$ \\
\hline Butyrate & $2.1(0.3)$ & $6.0(0.6)$ & $1.4(0.2)$ & $<0.001$ \\
\hline
\end{tabular}

*ANOVA across the dietary groups.

†Animals housed four per cage and faecal output from each cage divided by 4 ; results are mean (SEM) of three cages.

$\ddagger$ Mean (SEM) of 12 rats.

Table 2 Effect of diet on epithelial kinetic indexes in distal colonic epithelium

\begin{tabular}{lllll}
\hline & No fibre & Wheat bran & Methylcellulose & p Value * \\
\hline Crypt column height (cells) & $30.5(0.6) \dagger$ & $39.2(0.8)$ & $31.6(0.8)$ & $<0.001$ \\
No of metaphase arrests per crypt column & $1.1(0.2)$ & $1.9(0.3)$ & $1.0(0.3)$ & 0.026 \\
Mitotic index (\%) & $3.7(0.5)$ & $4.7(0.8)$ & $3.4(0.7)$ & 0.38 \\
\hline
\end{tabular}

*ANOVA across dietary groups.

†Mean (SEM) of 12 rats.

EFFECT OF DIET ON FAECAL AND CAECAL

INDEXES

The effects of diet on faecal and caecal indexes are shown in table 1 . Twenty four hour faecal output, measured at the end of the fourth week, was considerably higher in the wheat bran and methylcellulose fed animals compared with those fed no fibre. Significant differences in faecal and caecal $\mathrm{pH}$ were observed across the dietary groups and these were due primarily to the lower $\mathrm{pH}$ in animals fed wheat bran. Total faecal and caecal SCFA concentrations were highest in animals fed wheat bran and lowest in those fed methylcellulose. Butyrate concentrations followed the same pattern (table 1) as did

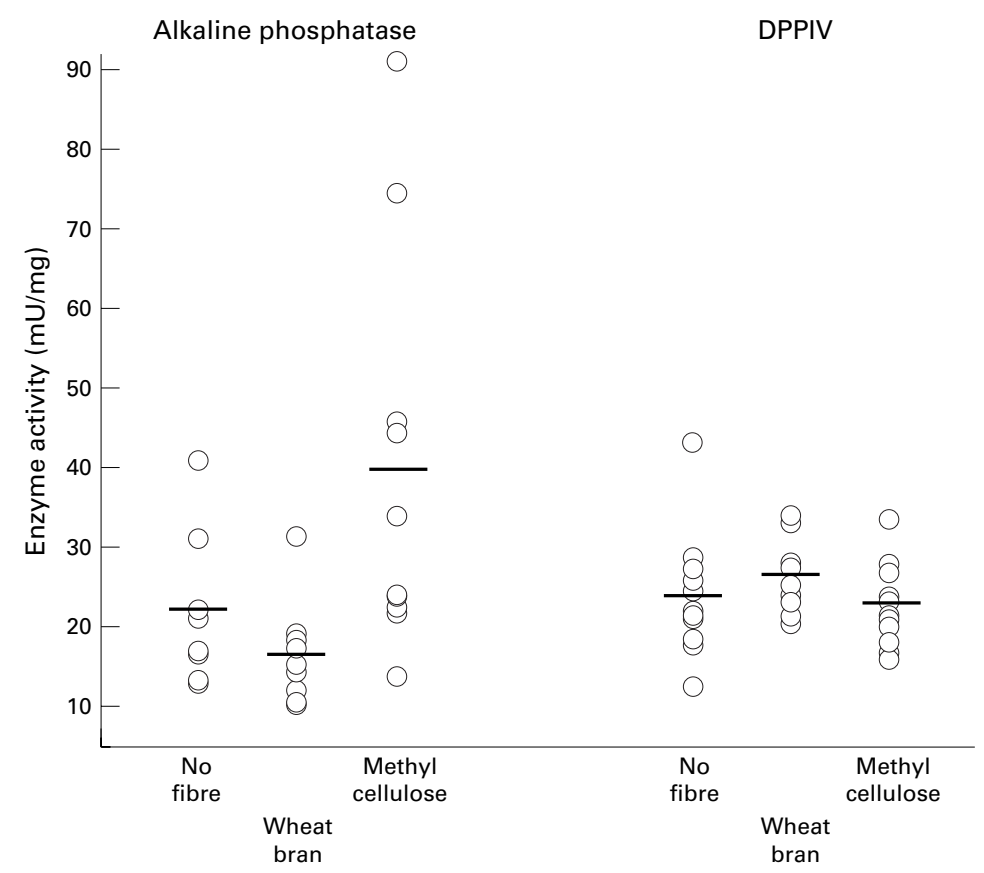

Figure 3 Effect of diet on brush border hydrolase activities of distal colonic mucosa. The diets contained no fibre, wheat bran, or methylcellulose. Alkaline phosphatase and dipeptidylpeptidase IV (DPPIV) activities differed significantly across the dietary groups $(p<0.001$ and $p<0.05$ respectively, ANOVA). Alkaline phosphatase activity in the methylcellulose group and DPPIV activity in the wheat bran group were significantly different to those in the other groups on multiple comparisons $(p<0.05)$. concentrations of acetate and propionate (data not shown).

EFFECT OF DIET ON MUCOSAL CHARACTERISTICS IN THE DISTAL COLON

Morphology

There was no evidence of epithelial cell damage or erosions, or of abnormalities in crypt architecture in any dietary group. Likewise, no histological evidence of mucosal inflammation was seen.

\section{Epithelial kinetics}

Table 2 shows the effects of diets on epithelial kinetic indexes. Diet significantly altered crypt column height $(\mathrm{CCH})$ in the distal colon $(\mathrm{p}<0.0001)$. Multiple comparisons showed that $\mathrm{CCH}$ was significantly increased in the wheat bran and methylcellulose fed animals compared with those fed no fibre $(p<0.05)$. $\mathrm{CCH}$ was also significantly higher in the wheat bran fed animals compared with those fed methylcellulose $(p<0.05)$. Statistically significant differences in the number of mitoses per colonic crypt column were also found across the dietary groups $(\mathrm{p}<0.05)$, due primarily to the increase induced by wheat bran. However, the mitotic index was similar across the groups.

\section{Activities of brush border hydrolases}

Significant differences were found across the dietary groups in mucosal alkaline phosphatase activities in the distal colon ( $p<0.001$; fig 3 ). Mean alkaline phosphatase activities in the methylcellulose fed animals were $243 \%$ and $180 \%$ higher than in the no fibre and wheat bran groups, respectively, and were responsible for this difference. DPPIV activities also significantly differed across the dietary groups, but this was due to higher activities in the wheat bran group (fig 3). The activities of alkaline phosphatase and DPPIV were also measured in jejunal mucosa, but no differences across the dietary groups were observed (data not shown).

\section{Discussion}

This study shows, for the first time, changes in distal colonic epithelial permeability induced by alterations of dietary intake in normal rats. Thus, ingestion of dietary fibre reduced transepithelial conductance and the apical to basolateral flux of ${ }^{51} \mathrm{Cr}$-EDTA by approximately $25 \%$ compared with ingestion of a no fibre diet for four weeks. EDTA, due to its hydrophilicity, permeates mainly via the paracellular route, indicating that the changes in permeability were due essentially to alterations in the paracellular pathway. A descending gradient of paracellular permeability from the jejunum to the colon has previously been reported using the techniques applied in the present study. ${ }^{19}$ In the rats fed fibre, such a gradient was evident; in rats fed no fibre, permeability in the distal colon was comparable with that in the jejunum. Diet induced differences in paracellular permeability were not observed in either the caecum or jejunum. Such localised sensitivity of the distal colon to the intake of dietary fibre has also been 
reported with regard to epithelial cell kinetics, as the effects of fibre on reversing epithelial atrophy are confined primarily to the distal colon. ${ }^{13} 23$

In an attempt to identify pathogenic mechanisms underlying diet induced changes in paracellular permeability, four aspects were considered. Firstly, the changes in permeability may have reflected epithelial erosion or ulceration associated with the lack of dietary fibre and not alterations in the function of tight junctions. Such a possibility was discounted, however, by the extensive morphological analysis of the distal colon, which showed no evidence of epithelial injury.

Secondly, the diets may have induced changes in permeability via mechanisms unrelated to colonic luminal and epithelial events. Small intestinal permeability may be influenced by deficiency of macronutrients ${ }^{4}$ or by stress. ${ }^{24}$ However, there was no evidence of such events occurring in the rats. The diets contained the recommended micronutrients and each diet was identical in that regard. Although 24 hour food intake was greater in fibre fed animals, this was expected as the fibre supplemented diets have a lower energy density. The rats gained weight to a similar degree across the three groups, and at no time showed evidence of stress.

Thirdly, associations between changes in luminal characteristics and paracellular permeability were investigated. Previous observations in Caco-2 monolayers suggested that SCFAs are key effectors in reducing paracellular permeability in vitro. ${ }^{10}$ Consistent with that previously reported, ${ }^{12}$ the wheat bran diet was associated with high levels of SCFAs in the faeces (an accurate indicator of the distal colonic environment ${ }^{12}$ ) and caecum. However, as also previously reported, ${ }^{13}$ methylcellulose caused very low concentrations of SCFAs in the faecal and caecal contents, levels that were even lower than those observed in rats fed the no fibre diet. In parallel with SCFA concentrations, $\mathrm{pH}$ was lower in the faecal and caecal contents of wheat bran fed animals than in the other two groups. As no correlation was found between the indexes of fermentation measured and paracellular permeability in either the distal colon or caecum, the hypothesis that SCFAs were responsible for improved barrier function is not supported.

Both wheat bran and methylcellulose exert a notable bulking effect on luminal contents. As we have previously found, ${ }^{12}{ }^{13}$ faecal output in animals fed the fibre supplemented diets was approximately 10 -fold that of the no fibre fed controls. The effects of wheat bran and methylcellulose on reducing paracellular permeability, therefore, paralleled their effects on faecal bulking. Fibre has previously been suggested to modulate colonic epithelial proliferation through its bulking effect, either by causing mechanical distension, ${ }^{25}$ or by causing surface cell abrasion. ${ }^{26}$ Other studies, however, have also shown that bulk alone is insufficient to modulate colonic epithelial cell proliferation, ${ }^{27} 28$ suggesting that fibres may have other properties that are fundamental to their effect.

Finally, the possibility that alterations in permeability reflected alterations in epithelial turnover and/or differentiation was addressed. Epithelial atrophy itself has been suggested to impair barrier function, ${ }^{9}$ and both wheat bran and methylcellulose have been shown to reverse such atrophy. ${ }^{13}$ Even though methylcellulose exhibited only mild (but statistically significant) effects in the present study, it is possible that small changes in $\mathrm{CCH}$ may have large effects on paracellular permeability. Rather than the number of cells in the epithelium, the maturity of the tight junctions is more likely to be a central factor governing paracellular permeability. Morphological studies have shown well formed tight junctions between surface epithelial cells in comparison to poorly formed tight junctions in cells deep in the crypt, ${ }^{11} 2930$ and immunohistochemical studies have shown that the tight junction proteins, ZO-1 and occludin, are predominantly expressed in the surface epithelium (Mariadason et al, manuscript in preparation). These observations suggest that the differentiated surface epithelium is largely responsible for paracellular barrier function of the epithelium. Furthermore, the morphological observations are in keeping with studies of both spontaneous and butyrate induced differentiation in Caco-2 cells, which suggest that the maturation of tight junctions is part of the differentiation process. ${ }^{1031}$ In the present study, measurement of the activities of brush border hydrolases in the mucosa was used to assess the differentiation status of the epithelium. ${ }^{32}{ }^{33}$ This technique has been successful in, for example, identifying the loss of surface epithelial cells in colonic mucosa in Ussing chambers exposed to $10 \mathrm{mM}$ butyrate (Mariadason et al, manuscript submitted). Both fibres significantly increased the activities of one hydrolase, DPPIV with wheat bran and alkaline phosphatase with methylcellulose. These different responses may each represent promotion of epithelial differentiation, although of differing phenotype. Studies in models of colonic epithelium in vitro, such as Caco-2 cell monolayers, have clearly shown that different stimuli can induce morphologically and functionally differentiated cells which exhibit different profiles of phenotypic markers of differentiation. ${ }^{34}{ }^{35}$

The contrasting spectra of effects that the two fibres exert on epithelial kinetics and mucosal hydrolase activities raise concerns that the search for a single mechanism of action in altering paracellular permeability may be too simplified, and are more suggestive that separate mechanisms are at work. Wheat bran, for example, may be working predominantly via supply of SCFAs. It is known that butyrate or SCFAs, when infused into the colonic lumen, can totally mimic the trophic effect of wheat bran. ${ }^{36}$ Butyrate and SCFAs also promote differentiation and improve barrier function in a colonic epithelial model system. ${ }^{10}$ Methylcellulose may be acting via an unknown mechanism to correct, at least partly, the atrophy and to promote cell differentiation. One 
possibility is that they represent the surface effect of the large amount of residual fibre in the lumen. Such hypotheses can only be addressed by further experimentation addressing the effect on barrier function of, for example, feeding inert bulking materials, or infusing SCFAs into the lumen of the colon.

Although a statistically significant difference in paracellular permeability was found across the groups, the biological significance of the observation is less easily ascertained. The rats in the present study did not suffer illness and there was no histological evidence of mucosal inflammation. However, rats are relatively resistant to the development of mucosal inflammation as exemplified by the lack of colitis in diverted colons, a feature that is invariable in the human equivalent. ${ }^{38}$ Clinical studies in situations where compromised barrier function plays a pathogenic role (such as in the critically ill patient or in ulcerative colitis) may be the best way of determining whether the dietary changes to permeability are indeed clinically relevant.

In conclusion, this study shows that the amount of fibre in the diet significantly alters barrier function by reducing paracellular permeability in the distal colon, but not the caecum or jejunum of normal rats. Features shared by the fibres include faecal bulking, a trophic effect on the colonic epithelium, and promotion of at least one feature of colonic epithelial differentiation. Because of the differences in the spectra of effects the two fibres exert on luminal indexes, epithelial kinetics, and mucosal hydrolase activities, it is proposed that they are acting to reduce paracellular permeability by different mechanisms.

JMM was in receipt of a Postgraduate Scholarship from the Faculty of Medicine, Dentistry and Allied Health Sciences, the University of Melbourne.

1 Almer S, Franzen L, Olaison G, et al. Increased absorption of polyethylene glycol 600 deposited in the colon in active ulcerative colitis. Gut 1993;34:509-13.

2 Walker WA, Sanderson IR. Epithelial barrier function to antigens. An overview. Ann N Y Acad Sci 1992;664:10-17. antigens. An overview. Ann N Y Acad Sci 1992;664:10-17.
Hermiston ML, Gordon GI. Inflammatory bowel disease and adenomas in mice expressing a dominant negative and adenomas in mice expressing a

4 Van Der Hulst RRWJ, Van Kreel BK, Von Meyenfeldt MF, et al. Glutamine and the preservation of gut integrity. Lancet 1993;341:1363-5.

5 Sandowski DC, Meddings JB. Luminal nutrients alter tightjunction permeability in the rat jejunum: an in vivo perfusion model. Can f Physiol Pharmacol 1993;71:835-9.

6 Iiboshi Y, Nezu R, Kennedy M, et al. Total parenteral nutrition decreases luminal mucous gel and increases permeability of small intestine. $\mathcal{F}$ Parenter Enteral Nutr 1994;18: 346-50.

7 Sundqvist T, Lindstrom F, Magnusson KE, et al. Influence of fasting on intestinal permeability and disease activity in patients with rheumatoid arthritis. Scand $\mathcal{f}$ Rheumato 1982;11:33-8.

8 Gibson PR. Ulcerative colitis: an epithelial disease? Baillieres Clin Gastroenterol 1997;11:17-33.

9 Wang Q, Xiang-dong W, Jeppsson B, et al. Influence of colostomy on in vivo and in vitro permeability of the rat colostomy on in vivo and in vitro perme
colon. Dis Colon Rectum 1996;39:663-70.

10 Mariadason JM, Barkla DH, Gibson PR. Effect of short-chain fatty acids on paracellular permeability in Caco-2 intestinal epithelium model. Am $\mathcal{F}$ Physiol 1997; 272:G705-12.
11 Luciano L, Reale E, Rechkemmer G, et al. Structure of zonulae occludentes and the permeability of the epithelium to short-chain fatty acids in the proximal and distal colon of guinea pig. 7 Membr Biol 1984;82:145-56.

12 McIntyre A, Young GP, Taranto T, et al. Different fibers have different regional effects on luminal contents of rat colon. Gastroenterology 1991;101:1274-81.

13 Folino M, McIntyre A, Young GP. Dietary fibers differ in their effects on large bowel epithelial proliferation and fecal fermentation-dependent events in rats. 7 Nutr 1995;125: 1521-8.

14 Young GP, McIntyre A, Albert V, et al. Wheat bran suppresses potato-starch potentiated colorectal tumorigenesis at the aberrant crypt stage in a rat model. Gastroenterology 1996;110:508-14.

15 McIntyre A, Gibson PR, Young GP. Butyrate production from dietary fibre and protection against large bowel cancer in a rat model. Gut 1993;34:386-91.

16 D'Argenio G, Cosenza V, Delle Cave M, et al. Butyrate enemas in experimental colitis and protection against large bowel cancer in a rat model. Gastroenterology 1996;110: 1727-34.

17 Medina V, Afonso JT, Alverez-Arguelles H, et al. Sodium butyrate inhibits carcinoma development in a $1,2-$
dimethylhydrazine-induced rat colon cancer. $f$ Parenter dimethylhydrazine-induced rat

18 Reeves PG. AIN-76 diet: Should we change the formulation? F Nutr 1989;119:1081-2.

19 Dawson DC. Intestinal absorption and secretion. In: Schultz SG, Field M, Frizzel RA, eds. Principles of membrane transport. Handbook of physiology. Bethesda: American Physiological Society, 1991:1-44.

20 Young GP, Rose IS, Cropper S, et al. Hepatic clearance of rat plasma intestinal alkaline phosphatase. Am $\mathcal{F}$ Physiol 1984;247:G419-26.

21 Maroux S, Louvard D, Baratti J. The aminopeptidase from hog intestinal brush border. Biochem Biophys Acta 1973; 321:282-95.

22 Bradford M. A rapid and sensitive method for the quantitation of microgram quantities of protein utilizing the principle of protein dye binding. Anal Biochem 1976;72:248-54.

23 Jacobs LR, Schneeman BO. Effects of dietary wheat bran on rat colonic structure and mucosal cell growth. F Nutr 1981; 111:798-803.

24 Saunders PR, Kosecka U, McKay DM, et al. Acute stressors stimulate ion secretion and increase epithelial permeability in rat intestine. Am ₹ Physiol 1994;267:G794-9.

25 Burnham D. Epithelial cell production and mucosal morphology in colonic obstruction. Cell Tissue Res 1983; 230:185-96.

26 Tasman-Jones C, Owens RL, Jones AL. Semipurified dietary fiber and small bowel morphology in rats. Dig Dis Sci 1982;27:519-24.

27 Dowling RH, Riecken EO, Laws JW, et al. The intestinal response to high bulk feeding in the rat. Clin Sci 1967;32:1-9.

28 Stragand JJ, Hagemann RF. Dietary influence on colonic cell renewal. Am f Clin Nutr 1977;30:918-23.

29 Madara JL, Trier JS, Neutra MR. Structural changes in the plasma membrane accompanying differentiation of epithelial cells in human and monkey small intestine. Gastroenterology 1980;78:963-75.

30 Marcial MA, Carlson SL, Madara JL. Partitioning of paracellular conductance along the ileal crypt-villus axis: a hypothesis based on structural analysis with detailed consideration of tight junction structure-function relationships. F Membr Biol 1984;80:59-70.

31 Pinto M, Robine-Leon S, Appay MD, et al. Enterocyte like differentiation and polarisation of the human colon carcinoma cell line Caco-2 in culture. Biol Cell 1983;47: 323-30.

32 Lev R, Griffiths WC. Colonic and small intestinal alkaline phosphatase. Gastroenterology 1982;82:1427-35.

33 Young GP, Macrae FA, Gibson PR, et al. Brush border hydrolases in normal and neoplastic colonic epithelium. $f$ Gastroenterol Hepatol 1992;7:347-54.

34 Basson MD, Hong F, Emenaker NJ. Specific modulation of intestinal epithelial brush border enzyme expression by a phorbol ester. F Surg Res 1995;59:121-6.

35 Halline AG, Davidson NO, Skarosi SF, et al. Effects of 1,25dihydroxyvitamin $\mathrm{D}_{3}$ on proliferation and differentiation of Caco-2 cells. Endocrinology 1994;134:1710-17.

36 Kripke SA, Fox AD, Berman JM, et al. Stimulation of intestinal mucosal growth with intracolonic infusion of shortchain fatty acids. F Parenter Enteral Nutr 1989;13:109-16.

37 Tang CL, Fielding M, Tjandra JJ, et al. A novel long-term caecal intubation model in the rat - effects of butyrate on epithelial kinetics [abstract]. $\mathcal{F}$ Gastroenterol Hepatol 1997; 12(suppl):A13.

38 Glotzer DJ, Glick ME, Goldman H. Proctitis and colitis following diversion of the fecal stream. Gastroenterology 1981; 80:438-41. 